\title{
Africa yields two full human genomes
}

To the growing list of people with fully sequenced genomes, two memorable names have now been added: Archbishop Desmond Tutu, the South African civilrights activist, and !Gubi, a Namibian hunter-gatherer.

!Gubi hails from the Khoisan community, one of the most ancient and diverse human populations. His is the first genome from a minority population in Africa to be sequenced. Comparing his sequence with the partial genome sequences of three other Khoisan shows that they are as different from one another as a European would be from an Asian, says team leader Stephan Schuster, a genome researcher at Pennsylvania State University in University Park. "This is despite the fact that they sometimes live within walking distance of one another," he adds.

These sequences are published today in Nature (see page 943) along with Tutu's, whose DNA was sequenced as a point of comparison because he comes from the Bantu, the majority population in southern Africa. The data could provide the tools needed to tackle some of the most fundamental questions of human evolution: where did modern humans originate in Africa? How long ago? When did the original migrations out of Africa occur, and who were the migrants?

"This information will help us to understand not only the diversity we see now, but also to trace how humans have evolved and accumulated these genetic differences," says David Comas, who studies human diversity at Pompeu Fabra University in Barcelona, Spain.

For Africans, the data might have more tangible benefits. The continent's rich genetic diversity means that some drugs designed to treat Europeans do not work well in Africans. And genome-wide association studies designed to trace the genetic underpinnings of disease are difficult to execute in Africa because the arrays used to detect genetic variation were designed using mostly European sequences. As a result, researchers lack the tools to study important medical mysteries, such as why the Khoisan and some other African populations are particularly susceptible to tuberculosis.

To date, none of the large human

\section{"We've been pushing for many years for the importance of studying diverse African populations."}

!Gubi's and Tutu's genomes each carry more than one million single base-pair changes that are not found in each other or in any of the published genomes, including one from a Yoruba individual from West Africa.

Several of the DNA changes in the Khoisan may reflect adaptations to the rigorous life of a hunter-gatherer in the Kalahari Desert. Three of the Khoisan carry a version of the muscle-expressed ACTN3 gene linked to faster sprinting. !Gubi carries one copy of a form of the $C L C N K B$ gene that encodes a cell-membrane channel capable of reabsorbing chloride ions in the kidney, a possible advantage in the desert. And
SILICON WHISKERS CATCH SUN'S RAYS

Tiny rods could make solar cells cheaper.

go.nature.com/1DRrnJ genome projects has focused on minority populations. "We've been pushing for many African populations," says Sarah Tishkoff, a genetic anthropologist at the University exciting to see this has now been done."

he Children's Cancer ical Research in Randwick, New South They selected four for further analysis, Y chromosome and focusing most divergent sequences. sequenced completely, and the full complement of proteincoding genes was sequenced from the other three Khoisan. all have a form of the taste-receptor gene TAS2R38 that would enable them to taste the bitter compound phenylthiocarbamide. This form of the gene may help hunter-gatherers to avoid toxic plants.

The Khoisan also lack a gene variant giving protection against malaria infection by Plasmodium vivax. This could reflect their lifestyle, Schuster says: with both water and cattle scarce, the Khoisan face little threat from malaria. But as agricultural communities encroach on the Khoisan habitat, or more Khoisan take up farming, the population may find itself increasingly vulnerable to infection.

Nevertheless, Tishkoff cautions against reading too much into the sequence data at this early stage. "You really need to have population-level information," she says. "If I see a variant in just one person, I don't know how prominent it's going to be in the rest of the population." To that end, Schuster and his team have used the sequence information to generate arrays containing more than 1.5 million markers that can be used to quickly assess genetic variation in large groups of people. They have already recruited hundreds of volunteers from different ethnic groups in Africa, and plan to begin using the arrays shortly.

Meanwhile, Tishkoff notes that the falling price and accelerating pace of sequencing mean that other African genomes are likely to be sequenced in the future. "We don't want to see any population being left out of this genomics revolution," she says. Heidi Ledford 